\title{
Muscle damage in response to a single bout of high intensity concentric exercise in patients with Pompe disease
}

\author{
Márk Váczi ${ }^{1}$, Szilvia Anett Nagy ${ }^{2,3,4,5}$, Tamás Kőszegi ${ }^{6}$, Bernadette Misovics ${ }^{7}$, Edina Szabó ${ }^{8}$, Éva Müller ${ }^{9}$, \\ Gábor Perlaki $^{2,4,5}$, Gergely Orsi ${ }^{2,4,5}$, József Pál ${ }^{10}$, Péter Bogner ${ }^{9}$, Zsolt Illes ${ }^{11,12,13}$ \\ ${ }^{1}$ Institute of Sport Sciences and Physical Education, University of Pécs, Pécs, Hungary; ${ }^{2}$ MTA-PTE Clinical Neuroscience MR Research Group, \\ Pécs, Hungary; ${ }^{3}$ Neurobiology of Stress Research Group, Szentágothai Research Center, University of Pécs, Pécs, Hungary; ${ }^{4}$ Department of \\ Neurosurgery, Medical School, University of Pécs, Pécs, Hungary; ${ }^{5}$ Pécs Diagnostic Centre, Pécs, Hungary; ${ }^{6}$ Department of Laboratory Medicine, \\ University of Pécs, Pécs, Hungary; ${ }^{7}$ University of Physical Education, Budapest, Hungary; ${ }^{8}$ Department of Anatomy, Medical School, MTA-PTE \\ PACAP Research Team, University of Pécs, Pécs, Hungary; ${ }^{9}$ Department of Medical Imaging, Medical School, University of Pécs, Pécs, Hungary; \\ ${ }^{10}$ Institute of Physiology, Medical School, University of Pécs, Pécs, Hungary; ${ }^{11}$ Department of Neurology, Medical School, University of Pécs, Pécs, \\ Hungary; ${ }^{12}$ Department of Neurology, Odense University Hospital, Odense, Denmark; ${ }^{13}$ Department of Clinical Research, University of Southern \\ Denmark, Odense, Denmark \\ Contributions: (I) Conception and design: M Váczi, SA Nagy, T Kőszegi, É Müller, J Pál, P Bogner, Z Illes; (II) Administrative support: SA Nagy, G \\ Perlaki, G Orsi, P Bogner, Z Illes; (III) Provision of study materials or patients: SA Nagy, G Perlaki, G Orsi, P Bogner, Z Illes, É Müller, J Pál; (IV) \\ Collection and assembly of data: M Váczi, SA Nagy, E Szabó, T Kőszegi, B Misovics; (V) Data analysis and interpretation: M Váczi, SA Nagy, E \\ Szabó, T Kőszegi, B Misovics, Z Illes; (VI) Manuscript writing: All authors; (VII) Final approval of manuscript: All authors. \\ Correspondence to: Márk Váczi. Institute of Sport Sciences and Physical Education, University of Pécs, Pécs, Hungary. Email: vaczi@gamma.ttk.pte.hu; \\ Zsolt Illes. Department of Neurology, University of Southern Denmark, Odense, Denmark. Email: zsolt.illes@rsyd.dk.
}

Background: In Pompe disease, resistance exercise could be an effective treatment to delay motor function impairment, however, the acute effects of this exercise modality are unclear.

Methods: In a prospective cohort study, we compared responses to a single bout of resistance exercise by serum markers of muscle damage and quantitative muscle magnetic resonance imaging (MRI) in patients $(n=12)$ and age- and gender-matched healthy controls $(n=12)$. Participants performed 50 maximal effort concentric knee flexions on a dynamometer.

Results: Twenty-four hours after exercise, levels of serum creatine kinase, lactate dehydrogenase and myoglobin increased in controls. In contrast, only myoglobin level increased in patients. All elevated serum markers declined by 48 hours after exercise in both groups. Mild soreness developed at 24 hours, which disappeared at 48 hours in both groups. In controls, MRI R2* relaxation rate reduced immediately and 24 hours after exercise, indicating increased water content and muscle perfusion. In patients, exercise had no effect on R2* values. The resistance exercise did not induce acute strength deficit in patients, rather, patients increased their strength by 24 hours. When serum marker changes were normalized to the magnitude of knee flexor tension developed during exercise, lactate dehydrogenase response was greater in patients.

Conclusions: Late-onset Pompe disease did not exacerbate exercise-induced muscle damage, however, lactate dehydrogenase may be monitored to screen high responders during high intensity resistance exercise interventions.

Keywords: Hamstring muscle; dynamometer; muscle microinjury; muscle magnetic resonance imaging (muscle MRI); Pompe disease

Submitted Apr 05, 2020. Accepted for publication Dec 16, 2020.

doi: 10.21037/atm-20-3114

View this article at: http://dx.doi.org/10.21037/atm-20-3114 


\section{Introduction}

Late-onset Pompe disease (LOPD) is a rare inherited metabolic myopathy with deficiency of acid alphaglucosidase (GAA) that results in glycogen accumulation in lysosomes. LOPD is characterized by a gradual decline in muscle size and strength, which eventually leads to impairments in motor function and mobility (1).

Enzyme replacement therapy (ERT) with recombinant human acid $\alpha$-glucosidase has been introduced and proved to be efficient in either stabilizing or improving strength and functional abilities (2). Exercise intervention as a potential add-on therapy has also been recommended in LOPD (3). Previous studies that applied combinations of aerobic and resistance exercise to avoid muscle wasting and maintain functionality, showed inconsistent results (3-5). This may primarily originate from moderate or uncontrolled exercise intensities, small sample size, uncontrolled experimental design, the lack of quantitative strength tests, and because exercise mode-specificity was not considered. The superiority of resistance versus aerobic exercise in the development of muscle strength has been shown in healthy humans regardless of gender, age, and training status (6). Furthermore, the magnitude of strength gain is sensitive to the tension developed in muscles during a particular resistance exercise training (7). Therefore, highintensity/load resistance exercise could potentially improve muscle weakness in LOPD patients.

There are concerns about the safety of resistance exercise in LOPD, because in healthy humans resistive (especially eccentric) muscle contractions induce myofibrillar disruptions, elevation of muscle protein and enzyme levels in serum [creatine kinase (CK), lactate dehydrogenase (LDH), myoglobin $(\mathrm{Mb})]$, muscle soreness, and strength deficit, commonly named as direct and indirect markers of exercise-induced muscle damage (EIMD) (8). In healthy humans, changes in these markers usually peak at $24 \mathrm{~h}$ postexercise. Evidences indicate that EIMD is less severe, when muscle contractions are performed concentrically $(8,9)$.

Limited data are available, how resistance exercise changes EIMD markers in LOPD patients. Although serum CK activity was monitored in one study during a 24-month enzyme replacement therapy in two patients, no exercise was performed (10). In a 12 -week-long combined resistance and endurance exercise intervention, CK was measured every 2 weeks (5). However, measurements were taken at unspecified times with respect to the actual exercise bouts: yet CK changes are sensitive within 12 to 48 hours post- exercise (11).

In LOPD patients, aerobic or submaximal resistance exercise or their combinations are recommended and excessive resistance exercises are contraindicated $(12,13)$. Despite of these recommendations, no evidence-based consensus exists whether high force resistance exercise exacerbates muscle damage in LOPD patients. Information on the acute effects of such exercise modality is needed and would contribute to proper exercise prescription. EIMD is considered as a normal response after high intensity unaccustomed exercise in healthy humans, which is moderate after concentric muscle contractions, but the responsiveness of LOPD patients is unknown. A wellknown technique to measure acute muscle responsiveness to exercise is quantitative magnetic resonance imaging (MRI) (14). Besides the most commonly used metrics (T1 and $\mathrm{T} 2$ relaxation times), previous studies have shown that $\mathrm{T}^{*}$ relaxation time may be sensitive to pathological tissue changes and functional impairment in skeletal muscles after exercise (15). Although, T2* relaxation time or R2* (R2* $\left.=1 / \mathrm{T} 2^{*}\right)$ relaxation rate is typically used to measure muscle perfusion after exercise, EIMD may affect R2* value due to magnetic field inhomogeneity caused by microtrauma.

Therefore, the purpose of the present study was to investigate the acute changes of serum and muscle MRI markers of EIMD in LOPD patients after maximal effort concentric contractions. We used isokinetic dynamometry to exercise and test the hamstring muscle, and compared the changes in LOPD patients to an age- and gender-matched healthy control group.

We present the following article in accordance with the STROBE reporting checklist (available at http://dx.doi. org/10.21037/atm-20-3114).

\section{Methods}

\section{Participants}

Seventeen Hungarian LOPD patients from different regions of Hungary were screened for eligibility in this prospective single-center cohort study, which was conducted during four consecutive years. Five patients were excluded because of the use of ventilation assistance, disability (wheelchair dependency), and acute or chronic knee pain, or their combination. Therefore, twelve LOPD patients were found eligible and enrolled. LOPD was confirmed in all patients by typical symptoms and reduced activity of acid alpha-glycosidase (GAA) in the blood. Previous exercise 
Table 1 Experimental design: investigation of the acute effects of high force concentric hamstring exercise on the indirect muscle damage markers

\begin{tabular}{lcccc}
\hline Variables in order of tests & Baseline & Immediately (post-exercise) & $24 \mathrm{~h}$ (post-exercise) & $48 \mathrm{~h}$ (post-exercise) \\
\hline $\mathrm{CK}, \mathrm{LDH}, \mathrm{Mb}$ & + & - & + & + \\
Muscle MRI & + & + & + \\
MVC torque & + & - & + \\
Muscle soreness & + & + & - \\
\hline
\end{tabular}

MVC, maximal voluntary contraction; CK, creatine kinase; LDH, lactate dehydrogenase; Mb, myoglobin; MRI, magnetic resonance imaging.

intervention studies demonstrated cases (3) or used smaller $(\mathrm{n}=5)$ samples (4). All patients (five men, seven women; mean age: $42.8 \pm 11.0$ years, age range: $19-58$ years, mean age at diagnosis: $38.2 \pm 9.9$ years, age range at diagnosis: 18-56 years) were treated according to a standardized scheme with $20 \mathrm{mg}$ alglucosidase alpha (Myozyme ${ }^{\circledR}$, SanofiGenzyme) per kilogram of body weight every 2 weeks. At the time of the investigation patients had been on ERT for $4.0 \pm 2.0$ years (range, $1-7$ years).

In order to reduce the number of confounding factors, age- and sex-matched healthy volunteers (five men, seven women; mean age: $42.7 \pm 11.2$ years, age range: 19 59 years), without a history of regular physical activity and any illness were also enrolled. Individuals were instructed to maintain their normal physical activity and to avoid any exercise one week before and during the study. The experimental protocol was approved by the Regional Ethics Committee (approval No. 4597) and performed in accordance with the ethical standards described in the provisions of the Declaration of Helsinki (as revised in 2013). Every participant was informed about the procedure and signed their written informed consent before the examination.

\section{Experimental design}

Patients were hospitalized for three days in the study center. The investigation consisted of three consecutive days (Table 1), and took place in a biomechanics laboratory (for strength tests and exercise) and in a diagnostic center (for MRI measurements and blood analyses). On day 1, first blood was taken for serum markers; then quantitative muscle MRI was performed followed by measurement of maximal voluntary contraction (MVC) torque and muscle soreness, and finally by dynamometric exercise. Immediately after exercise, another MVC and quantitative muscle
MRI were performed. On day 2 (at $24 \mathrm{~h}$ post-exercise), all measurements were repeated in the same order. On day 3 (at $48 \mathrm{~h}$ post-exercise), only serum markers and soreness were measured.

\section{Maximal voluntary contraction}

Before any strength evaluation, participants warmed-up by cycling for 5 minutes at a self-selected speed on a stationary cycle ergometer, and then by stretching the hamstring muscle. MVC torque of the hamstring was measured using Multicont II isokinetic device (Mediagnost Ltd, Budapest, and Mechatronic Ltd, Szeged, Hungary). Participants were seated on the dynamometer's seat. The shin of the tested lower extremity was fastened with a strap to the lever of the dynamometer above the ankle. Before any testing, gravitation compensation was performed for the tested leg to avoid inaccurate data collection originating from limb weight. Then three concentric knee flexions were executed between $10^{\circ}$ and $80^{\circ}$ range of motion, with 1 minute rest between repetitions. The threshold trigger torque in these contractions to initiate the movement was $5 \mathrm{Nm}$. As participants reached this torque the dynamometer rotated to permit the knee flexion, and participants had to flex their knee with maximal effort. The MVC's were performed at $60 \%$ constant angular velocity. Data giving the torque-time curve were exported, and average torque was calculated offline and was considered as MVC torque.

\section{Blood sampling and analyses}

Ten $\mathrm{ml}$ blood was drawn from an antecubital vein. After clotting, the blood sample was centrifuged at $1,500 \mathrm{~g}$ for $10 \mathrm{~min}$ to obtain serum. Serum samples were stored at $-70^{\circ} \mathrm{C}$ until being analysed. $\mathrm{CK}$ and $\mathrm{LDH}$ activities were determined by kinetic optimized UV method (COBAS 


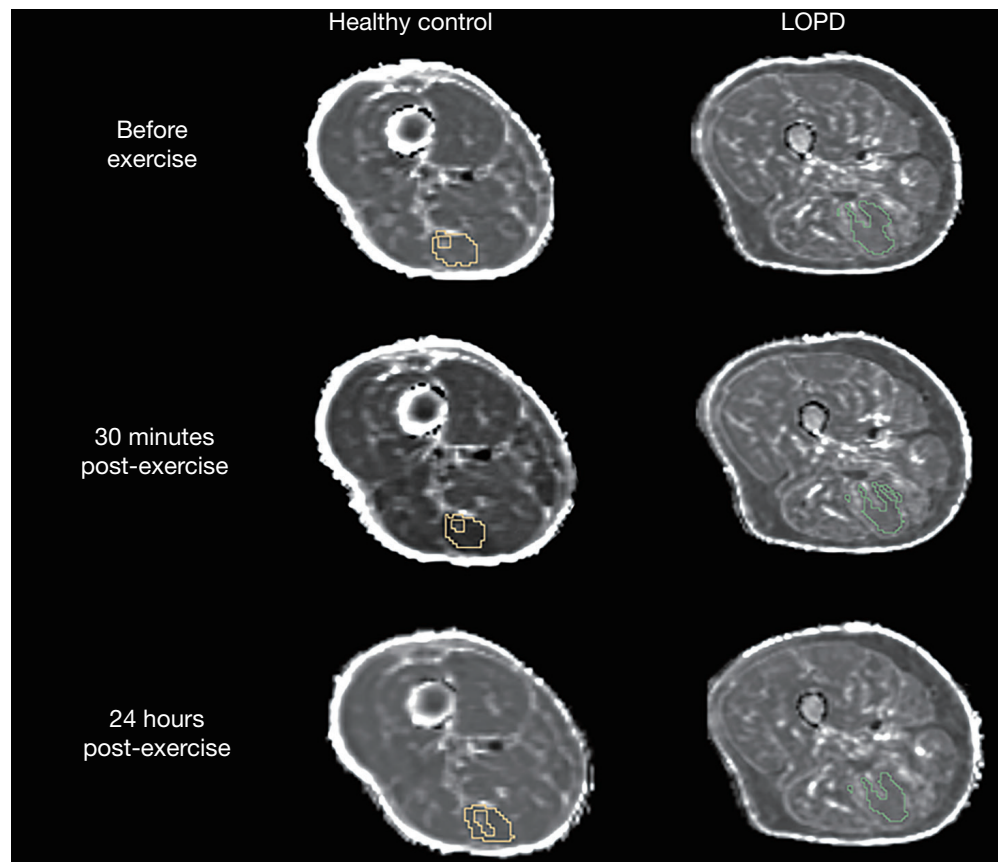

Figure 1 A representative image of muscle MRI (R2* map). Image of the semitendinosus muscle MRI (R2* map) in a patient with LOPD and in a healthy control are shown before and after exercise at different time points. LOPD, late-onset Pompe disease.

INTEGRA $^{\circledR} 400$ plus, Roche Diagnostics GmbH, Mannheim, Germany). The reference range was 0-200 IU/L for CK and 240-480 IU/L for LDH. Mb was measured by chemiluminescence automated immunoassay (Immulite 1000, Siemens Healthcare Diagnostics GmbH, Marburg, Germany). The upper reference limit at 97.5 percentile for $\mathrm{Mb}$ was $70 \mu \mathrm{g} / \mathrm{L}$ and the median value was $25 \mu \mathrm{g} / \mathrm{L}$.

\section{Magnetic resonance imaging}

Imaging was performed using a $3 \mathrm{~T}$ Magnetom TIM Trio whole-body MRI scanner (Siemens AG, Erlangen, Germany) with a body matrix coil combined with the spine matrix coil. For R2* mapping, a multi-echo 3D FLASH sequence with 10 equally spaced echoes $(\mathrm{TR} / \mathrm{TE} 1=36$ $/ 2.28 \mathrm{~ms}$; inter-echo spacing $=3.43 \mathrm{~ms}$; Flip Angle $=16^{\circ}$; 208 sagittal slices; slice thickness $=2 \mathrm{~mm} ; \mathrm{FOV}=250$ $\times 250 \mathrm{~mm}^{2}$; matrix size $=128 \times 128$; receiver bandwidth $=300 \mathrm{~Hz} /$ pixel) was used. The shortest echo time was limited by the resolution selected. Multi-echo 3D FLASH sequence was positioned according to the following: the top of the field of view was shifted from the right head of the femur distally to properly cover the hamstring muscles. This distance was measured individually and used during the repeated MR examinations to ensure appropriate repositioning. MRI examination of the hamstring complex was performed before exercise, $30 \mathrm{~min}$ and $24 \mathrm{~h}$ postexercise. MRI data analysis was performed only in 8 patients with LOPD and in their matched control subjects due to disease related intramuscular fatty infiltration.

Matlab $^{\circledR}$ and a self-written program code were used for quantitative MR data analysis (The Math Works, Inc., Natick, MA, USA). R2* maps were calculated by voxelwise nonlinear least-squares fitting of the mono-exponential signal decay over echo time for each voxel where the goodness of fit (r-square) was greater than 0.9 \{Eq. [1]\}.

$$
S_{T E}=S_{0} \times e^{\left(-T E \times R 2^{*}\right)}
$$

Hamstring muscles i.e., semitendinosus (ST) and semimembranosus (SM) muscles, and long head of biceps femoris (BF) were manually delineated (with FSL software, https://fsl.fmrib.ox.ac.uk/fsl/fslwiki/) by an experienced observer on R2* maps excluding subcutaneous and intermuscular fat, vessels, nerves, and fasciae (Figure 1). To increase the reliability, $\mathrm{R} 2 *$ maps of the three time points were linearly registered to each other using the FSL Linear Image Registration Tool Version 4.1 (16). R2* values were then derived separately from each muscle in the control 
group, while only ST muscle was evaluated in patients with LODP due to significant intramuscular fatty infiltration in $\mathrm{BF}$ and SM muscles.

\section{Delayed onset muscle soreness}

After performing the MVCs, subjects were asked to report their perceived delayed onset muscle soreness in the hamstring muscle. Soreness was assessed by a $50 \mathrm{~mm}$ visual analogue scale $(0=$ no pain, $50=$ very sore $)(17)$.

\section{Concentric hamstring exercise}

Subjects exercised the hamstring in a sitting position using the same dynamometer as described previously. The concentric contraction started at a knee angle of $10^{\circ}$ and ended at $80^{\circ}$. A trigger threshold of $5 \mathrm{Nm}$ was set for the contractions, which the participants had to achieve to initiate the dynamometer's lever arm to move into the direction of concentric contraction. The contraction velocity was $30^{\circ} \%$ s. Subjects were encouraged to maximally execute the knee flexions. When the lever reached the $80^{\circ}$ position, it automatically returned to the $30^{\circ}$ position with the same velocity, while subjects relaxed their muscles. There were 5 sets of 10 repetitions hamstring contractions with 2 minutes rest between sets. Average torque of each exercise contraction was calculated and further averaged in each set (set torque) and in the entire exercise (exercise torque) given by the five sets. Visual feedback on effort and verbal encouragement was given to participants during both the MVC tests and the exercise.

\section{Data analysis}

Data analyses were performed using IBM SPSS Statistics for Windows, Version 20.0 (IBM Corp., Armonk, NY, USA). Means and standard error of the means (SEM) were calculated. Between-group differences in the baseline values were determined using independent $t$-tests (in $\mathrm{LDH}$ ) or Mann-Whitney U-tests (in MVC torque, CK, Mb, and soreness), depending on the Shapiro-Wilk normality test results. Exercise torque was compared between groups using independent $t$-test.

Except R2*, we determined the exercise effect across time in every measured variable using nonparametric Friedman ANOVA. This was performed because of the results of the normality tests and/or because the baseline values were significantly different between groups. To test differences in means among the different test times, Wilcoxon Matched Pairs tests were used for post-hoc analysis. One-way repeated measures ANOVA was performed with a posthoc test including Bonferroni pairwise comparisons to compare changes in $\mathrm{R} 2{ }^{*}$ values among the time points. The assumptions of that were satisfied as judged by testing for normality, outliers, and sphericity.

Relative changes in serum markers and muscle soreness were also determined. This was performed by using the peak values, which were expressed as percent of the baseline values, except muscle soreness, in which the peak values were considered as relative change. The relative changes in the above variables were then normalized to exercise torque. This was necessary because the magnitude of muscle tension influences EIMD, and with this procedure we were able to differentiate group responses relative to their muscle tension, and to reduce the confounding effect of fitness status. Relative changes and normalized changes were compared between groups using Mann-Whitney $U$ tests. Results were considered significant at $\mathrm{P}<0.05$ for all statistical tests.

\section{Results}

All participants $(n=24)$ executed the measurement tests. There were neither dropouts nor missing data.

\section{Effect of concentric exercise on maximal voluntary contraction torque}

At baseline, MVC torque was significantly smaller in LOPD patients than in controls $(\mathrm{P}=0.0001)$. Exercise torque was significantly lower in LOPD patients $(53 \pm 5 \mathrm{Nm})$ compared to controls $(73 \pm 8 \mathrm{Nm})(\mathrm{P}=0.015)$.

Friedman ANOVA revealed significant time effect for MVC torque in both groups $(\mathrm{P}=0.0001$ for $\mathrm{LOPD}$ patients, $\mathrm{P}=0.003$ for controls) (Figure 2). The post-hoc analyses showed that MVC torque reduced significantly immediately after exercise $(\mathrm{P}=0.003)$, and then recovered to baseline at $24 \mathrm{~h}$ post-exercise in controls. The reduction in LOPD patients immediately after exercise only approached the significance level $(\mathrm{P}=0.06)$. However, MVC torque was greater at $24 \mathrm{~h}$ post-exercise compared to the baseline $(\mathrm{P}=0.028)$.

\section{Effect of concentric exercise on serum biomarkers of muscle microinjury}

All baseline serum biomarker mean levels were within the 

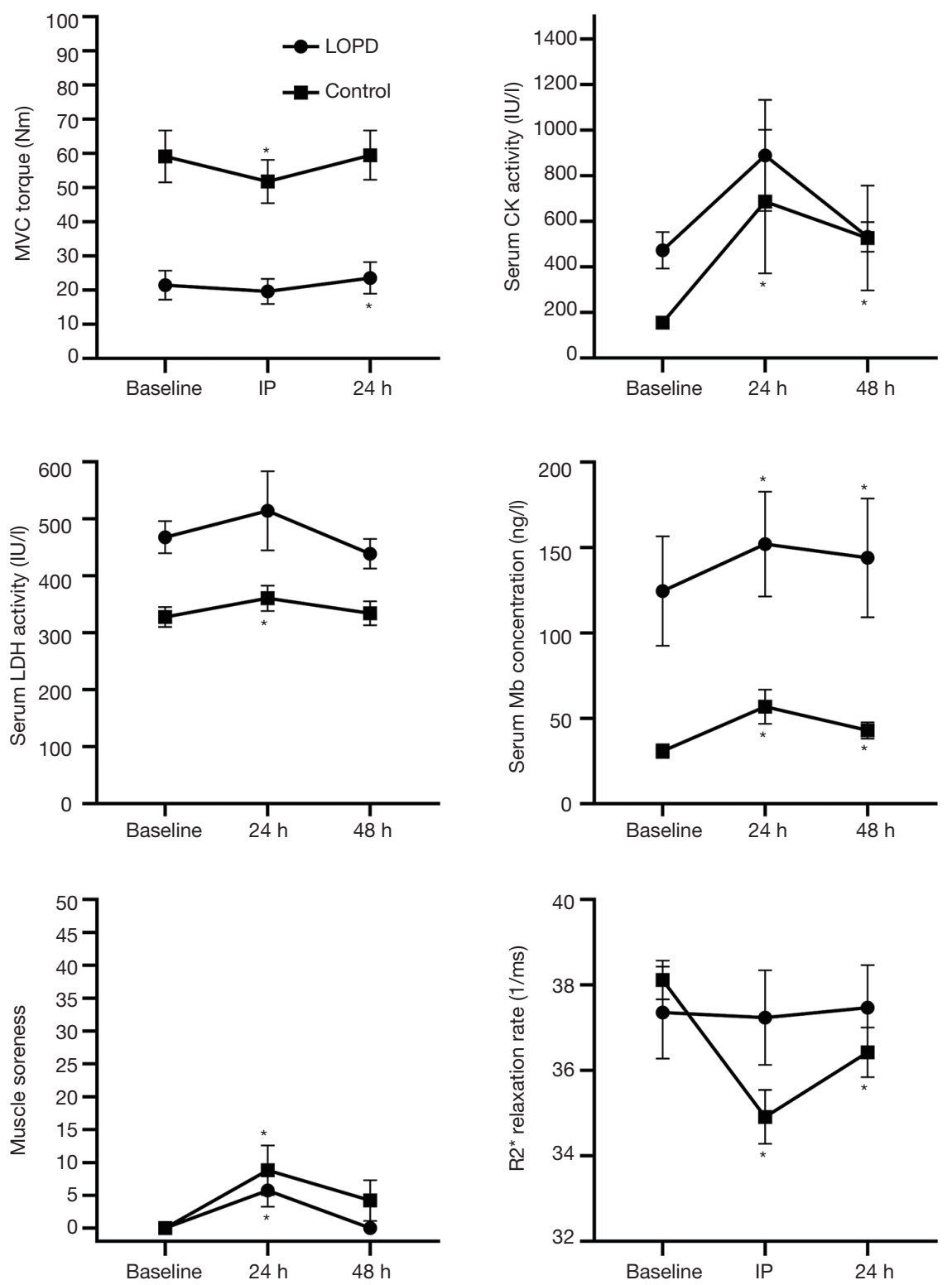

Figure 2 Acute effect of concentric hamstring exercise on indirect skeletal muscle damage indicators. Effects on MVC torque, serum biomarkers, muscle soreness and quantitative muscle MRI are shown in patients with LOPD and healthy controls. *, significant difference from baseline $(\mathrm{P}<0.05)$. mean \pm SEM is shown. IP, immediately post-exercise; $24 \mathrm{~h}, 24$ hours after exercise; MVC, maximal voluntary contraction; CK, serum creatine kinase activity; LDH, lactate dehydrogenase activity; Mb, serum myoglobin concentration; LOPD, lateonset Pompe disease.

normal reference range in both groups, though few outliers were found among LOPD patients (Figure 2). At baseline, levels of CK $(\mathrm{P}=0.0001)$, LDH $(\mathrm{P}=0.0001)$, and $\mathrm{Mb}$ $(\mathrm{P}=0.006)$ were significantly higher in LOPD patients than in controls.

In controls, all biomarker levels increased significantly at $24 \mathrm{~h}$ post-exercise $(\mathrm{P}<0.05)$, and then started to recover at $48 \mathrm{~h}$ post-exercise (Figure 2). However, this recovery was incomplete $(\mathrm{P}<0.05)$, except LDH activity. In LOPD patients, $\mathrm{Mb}$ increased significantly at $24 \mathrm{~h}(\mathrm{P}=0.010)$, and remained elevated at $48 \mathrm{~h}$ (Figure 2). The tendency of increase in the CK activity at $24 \mathrm{~h}$ only approached the 


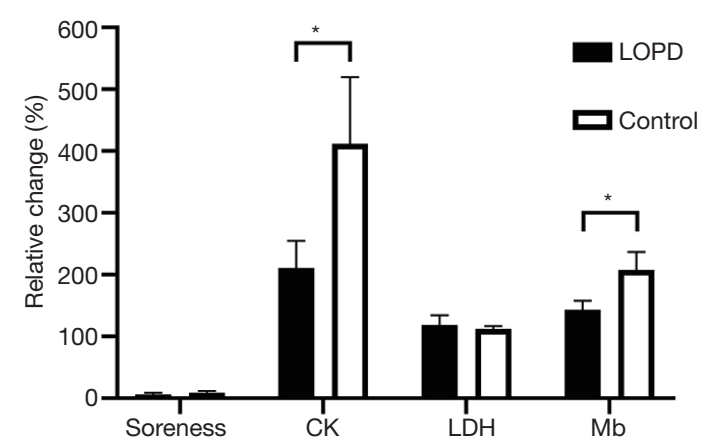

Figure 3 Exercise-induced relative changes in serum markers and hamstring soreness. Peak values as percent of the baseline values (mean \pm SEM) are shown. *, significant difference between groups $(\mathrm{P}<0.05)$.

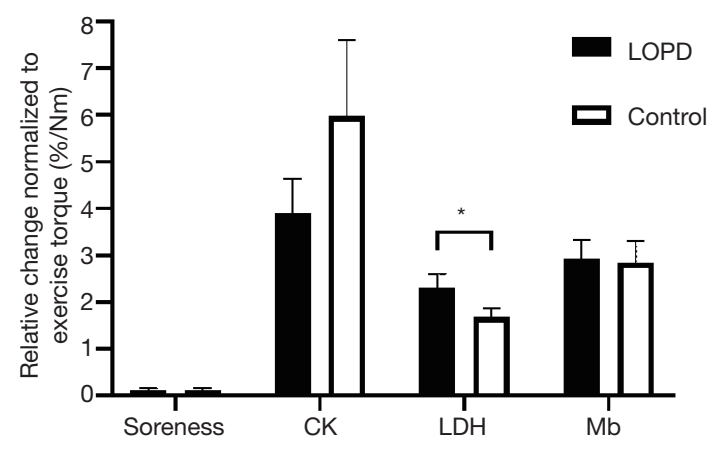

Figure 4 Exercise-induced changes in serum markers and hamstring soreness normalized to exercise torque. Mean \pm SEM are shown. *, significant difference between groups $(\mathrm{P}<0.05)$.

significance level $(\mathrm{P}=0.078)$ (Figure 2). The time effect in $\mathrm{LDH}$ was significant $(\mathrm{P}=0.020)$, however, the post-hoc test revealed only a significant reduction from 24 to $48 \mathrm{~h}$ postexercise $(\mathrm{P}=0.012)$ (Figure 2).

\section{Effect of concentric exercise on muscle soreness}

Exercise increased muscle soreness in both LOPD patients $(\mathrm{P}=0.007)$ and healthy controls $(\mathrm{P}=0.002)$ by 24 hours, which disappeared at $48 \mathrm{~h}$ post-exercise (Figure 2).

\section{Effect of concentric exercise on R2* values of muscle MRI}

Significant time effect was found for $\mathrm{R}^{*}$ values in the semitendinosus (ST) muscle of controls $\left\{\mathrm{F}_{[2,14]}=16.65\right.$; $\mathrm{P}=0.0002\}$. Post-hoc test revealed that in controls, concentric muscle exercise elicited a significant reduction in $\mathrm{R}^{*}$ values from baseline to IP $(\mathrm{P}=0.006)$ and $24 \mathrm{~h}$ postexercise $(\mathrm{P}=0.021)$. We observed a trend of recovery, i.e., increase by 24 hours, although it was not significant $(\mathrm{P}=0.072)$. $\mathrm{BF}$ and $\mathrm{SM}$ muscles did not show significant differences in R2* values over time in the control group. In patients with LOPD, concentric muscle exercise had no statistically significant effect on R2* values in the ST muscle (Figure 2).

\section{Change of biomarkers relative to exercise torque}

The relative change in $\mathrm{CK}$ and $\mathrm{Mb}$ concentration was significantly greater in healthy individuals than in LOPD patients $(\mathrm{P}=0.047$ and $\mathrm{P}=0.038$, respectively). $\mathrm{LDH}$ levels and muscle sore were not different (Figure 3). When the relative changes in serum markers and muscle soreness were normalized to exercise torque, $\mathrm{LDH}$ concentration was significantly higher in LOPD patients $(\mathrm{P}=0.039)$ (Figure 4).

\section{Discussion}

Here we used a complex experimental approach to examine muscle damage after a standardized single bout of high intensity concentric exercise in patients with LOPD. We measured serum biomarkers, MCV torque and quantitative muscle MRI repeatedly at different time-points after the standardized exercise, and compared the outcomes to controls at baseline and sequentially. We found that LOPD subjects did not show evidence of increased muscle damage compared to controls, as they showed similar or less severe responsiveness in EIMD markers, except LDH activity.

In healthy humans, tension developed in muscles induces myofibrillar and membrane ruptures, leading to elevations in serum enzymes and proteins, inflammation, and acute strength deficit $(9,17)$. These responses are considered normal, and microinjury could be an important signal in the induction of hypertrophic changes (18).

At baseline, few LOPD patients demonstrated abnormal $\mathrm{CK}, \mathrm{LDH}$, and $\mathrm{Mb}$ levels, though the mean values were within the normal reference range. Also, LOPD patients produced $65 \%$ less hamstring torque than the healthy controls. Slight elevations in serum markers and muscle wasting are in line with damage of muscle fibres resulting from glycogen accumulation $(11,12)$.

In the control group, all serum markers increased, muscle soreness developed and peaked 24 hours after exercise, similarly other healthy human groups (19). The greatest 
increase (4-fold) was found in CK activity, confirming to show the highest sensitivity (20). Smaller increase was found in LDH activity and Mb concentration, and their peak values remained within the normal reference range. Although serum marker changes and dynamics in LOPD were similar to the control group, only $\mathrm{Mb}$ concentration was significantly elevated. The lack of statistically significant changes in the other serum markers can be attributed to little tension during the exercise avoiding mechanical stress, and the high variability of responses due to the wide range of strength status. Furthermore, seven out of the twelve LOPD patients did not report muscle soreness at all at any time points, suggesting little or no inflammation caused by EIMD. Though soreness was reported during maximal effort contraction (a sensitive test), data still should be used with caution because soreness sometimes can be overlooked by patients.

Immediately after exercise, hamstring torque reduced to $85 \%$ of the baseline in the healthy group, while there was no change in LOPD. MVC torque represents the maximum contractility, and an immediate drop in MVC torque is commonly named as fatigue. For the acute fatigue in the control group, metabolic changes rather than EIMD might be responsible, considering the full recovery 24 hours later. The acute energy demand is high during pure concentric contraction, and metabolite accumulation is evident shortly after high intensity concentric exercise training $(21,22)$. Because LOPD patients performed less mechanical torque during exercise, the smaller rate of fatigue may be the result of the smaller number of active motor units requiring less energy, inducing less metabolic changes. Greater anaerobic capacity and the selective recruitment of the more fatigable fast fibres may also have led to greater fatigue in the controls. The immediate $\mathrm{R} 2$ * reduction in controls confirms this, i.e., increased water content and muscle perfusion could be the result of excessive anaerobic work, while in patients with LOPD absence of effect on R2* values suggested no significant alteration in water content, perfusion and muscle injury (15).

At 24 hours post-exercise, recovery, i.e., normal MVC torque was seen in the healthy group despite of elevation in biomarkers. In LOPD patients, torque also increased compared to baseline. Normal or even increased contractility after intensive exercise is not unique because early neural adaptation compensates for the acute strength deficit, when EIMD is less severe (23). The early strength recovery/ gain suggests increased neural drive to the muscle or the recruitment of new motor units, indicatives of exercise adaptation in both the controls and LOPD patients. The moderate responses and the quick ( $48 \mathrm{~h}$ ) recovery in the serum markers, regardless of health status, are explained by the fact that concentric exercise contractions with less mechanical stress were applied. We can conclude that although soreness and all serum markers show similar dynamics, healthy individuals show higher serum marker response sensitiveness, while LOPD patients demonstrate higher serum marker response variability, smaller acute fatigability, and better strength adaptability at $24 \mathrm{~h}$ postexercise.

Most previous studies used absolute level of markers to quantify EIMD $(24,25)$. We also compared the change in the serum markers normalized to the exercise torque, thus relative to the muscle tension developed in the hamstring muscle. We found that LDH normalized to exercise torque increased more in LOPD patients, and this might be the result of the low fitness status (26). $\mathrm{LDH}$ response is also influenced by muscle fiber type composition, which was uncontrolled in our study (27). It is notable, however, that one patient (outlier) responded with very high $\mathrm{LDH}$ activity $(1,155 \mathrm{IU} / \mathrm{L}$, more than two-fold of the group mean) at 24-h post-exercise. When we removed this data, the statistics showed non-significant change. High LDH responders may show less intracellular energy levels of the muscle fibres, and gene polymorphism of methylenetetrahydrofolate reductase (MTHFR) and paraoxonase 1 (PON1) enzymes might influence the exercise induced release of muscle biomarkers (28). Also, biomarker levels depend on their release kinetics (fastest for $\mathrm{Mb}$ ), and on the half-life of the tested parameters (LDH the longest).

Limitation of the present study is that we did not measure the hamstring myoelectric activity, therefore we do not know if the source of strength recovery/gain is neural. Furthermore, only the ST muscle could be evaluated by MRI in patients with LOPD due to intramuscular fatty infiltration. However, according to previous MRI studies, ST muscle showed the most significant exercise-induced muscle alterations in the hamstring complex, confirming high sensitiveness of the MRI test in our experiment $(29,30)$. Finally, though exercise-induced responses in our cohort of 12 patients were controlled with gender-and age-matched healthy participants, and were normalized to fitness level, the present data cannot be generalized to the LOPD population 
with severe alteration of muscle strength and ventilation.

\section{Conclusions}

In conclusion, considering the absolute changes in the serum markers, LOPD did not cause exacerbation of EIMD after a single bout of high intensity concentric exercise compared to controls. However, when the magnitudes of changes in the serum markers were normalized to exercise torque, the LDH response was greater in patients. Therefore, monitoring of LDH in the early phase of a systematic resistance exercise training intervention may help to decide about training effort, and submaximal (80-90\%) intensity resistance exercises may limit muscle damage in high LDH responders.

\section{Acknowledgments}

Funding: The study was supported by EU Social Funds (EFOP3.6.2-16-2017-00008, "The role of neuroinflammation in neurodegeneration: From molecules to clinics", and EFOP3.6.1.-16-2016-00004 Comprehensive development for implementing smart specialization strategies at the University of Pécs), the GINOP (2.3.2-15-2016-00047), and the Sanofi-Genzyme.

\section{Footnote}

Reporting Checklist: The authors have completed the STROBE reporting checklist. Available at http://dx.doi. org/10.21037/atm-20-3114

Data Sharing Statement: Available at http://dx.doi. org/10.21037/atm-20-3114

Peer Review File: Available at http://dx.doi.org/10.21037/ atm-20-3114

Conflicts of Interest: All authors have completed the ICMJE uniform disclosure form (available at http://dx.doi. org/10.21037/atm-20-3114). SAN reports grants from the New National Excellence Program of the Ministry of Human Capacities (ÚNKP-17-3-III-PTE-315) and a grant from the János Bolyai Research Scholarship of the Hungarian Academy of Sciences. MV reports grants from the Institutional Excellence Program, University of Pécs (178864/2018 FEKUTSTRAT) during the conduct of the study. The authors have no other conflicts of interest to declare.
Ethical Statement: The authors are accountable for all aspects of the work in ensuring that questions related to the accuracy or integrity of any part of the work are appropriately investigated and resolved. The experimental protocol was approved by the Regional Ethics Committee (approval no. 4597) and performed in accordance with the ethical standards described in the provisions of the Declaration of Helsinki (as revised in 2013). Every participant was informed about the procedure and signed their written informed consent before the examination.

Open Access Statement: This is an Open Access article distributed in accordance with the Creative Commons Attribution-NonCommercial-NoDerivs 4.0 International License (CC BY-NC-ND 4.0), which permits the noncommercial replication and distribution of the article with the strict proviso that no changes or edits are made and the original work is properly cited (including links to both the formal publication through the relevant DOI and the license). See: https://creativecommons.org/licenses/by-nc-nd/4.0/.

\section{References}

1. Van der Ploeg AT, Reuser AJJ. Pompe's disease. Lancet 2008;372:1342-53.

2. Schoser B, Stewart A, Kanters S, et al. Survival and longterm outcomes in late-onset Pompe disease following alglucosidase alfa treatment: a systematic review and metaanalysis. J Neurol 2017;264:621-30.

3. Krase AA, Papadimas GK, Terzis GD. Functional capacity in a late-onset Pompe disease patient: Effect of enzyme replacement therapy and exercise training. Ann Indian Acad Neurol 2016;19:423-4.

4. Terzis G, Dimopoulos F, Papadimas GK, et al. Effect of aerobic and resistance exercise training on late-onset Pompe disease patients receiving enzyme replacement therapy. Mol Genet Metab 2011;104:279-83.

5. van den Berg LEM, Favejee MM, Wens SCA, et al. Safety and efficacy of exercise training in adults with Pompe disease: evalution of endurance, muscle strength and core stability before and after a 12 week training program. Orphanet J Rare Dis 2015;10:87.

6. Nader GA. Concurrent strength and endurance training: from molecules to man. Med Sci Sports Exerc 2006;38:1965-70.

7. Schoenfeld BJ, Peterson MD, Ogborn D, et al. Effects of Low- vs. High-Load Resistance Training on Muscle Strength and Hypertrophy in Well-Trained Men. J 


\section{Page 10 of 10}

strength Cond Res 2015;29:2954-63.

8. Clarkson PM, Byrnes WC, McCormick KM, et al. Muscle soreness and serum creatine kinase activity following isometric, eccentric, and concentric exercise. Int J Sports Med 1986;7:152-5.

9. Nosaka K, Lavender A, Newton M, et al. Muscle Damage in Resistance Training. Int J Sport Heal Sci 2003;1:1-8.

10. Vielhaber S, Brejova A, Debska-Vielhaber G, et al. 24-months results in two adults with Pompe disease on enzyme replacement therapy. Clin Neurol Neurosurg 2011;113:350-7.

11. Cabannis CD. Clinical Methods: The History, Physical, and Laboratory Examinations. 3rd ed. In: Kenneth WH, Dallas HW, Hurst JW, editor. Boston: Butterworths, 1990:161-2.

12. Case LE, Kishnani PS. Physical therapy management of Pompe disease. Genet Med 2006;8:318-27.

13. Cupler EJ, Berger KI, Leshner RT, et al. Consensus treatment recommendations for late-onset Pompe disease. Muscle Nerve 2012;45:319-33.

14. Carlier PG, Marty B, Scheidegger O, et al. Skeletal Muscle Quantitative Nuclear Magnetic Resonance Imaging and Spectroscopy as an Outcome Measure for Clinical Trials. J Neuromuscul Dis 2016;3:1-28.

15. Varghese J, Scandling D, Joshi R, et al. Rapid assessment of quantitative $\mathrm{T} 1, \mathrm{~T} 2$ and $\mathrm{T} 2{ }^{*}$ in lower extremity muscles in response to maximal treadmill exercise. NMR Biomed 2015;28:998-1008.

16. Jenkinson M, Smith S. A global optimisation method for robust affine registration of brain images. Med Image Anal 2001;5:143-56.

17. Murayama M, Nosaka K, Yoneda T, et al. Changes in hardness of the human elbow flexor muscles after eccentric exercise. Eur J Appl Physiol 2000;82:361-7.

18. Schoenfeld BJ. Does exercise-induced muscle damage play a role in skeletal muscle hypertrophy? J strength Cond Res 2012;26:1441-53.

19. Váczi M, Tihanyi J, Hortobágyi T, et al. Mechanical, biochemical, and electromyographic responses to shortterm eccentric-concentric knee extensor training in humans. J strength Cond Res 2011;25:922-32.

Cite this article as: Váczi M, Nagy SA, Köszegi T, Misovics B, Szabó E, Müller É, Perlaki G, Orsi G, Pál J, Bogner P, Illes Z. Muscle damage in response to a single bout of high intensity concentric exercise in patients with Pompe disease. Ann Transl Med 2021;9(5):389. doi: 10.21037/atm-20-3114
Váczi et al. Acute resistance exercise effects in Pompe disease

20. Brancaccio P, Maffulli N, Buonauro R, et al. Serum enzyme monitoring in sports medicine. Clin Sports Med 2008;27:1-18, vii.

21. Tesch PA, Dudley GA, Duvoisin MR, et al. Force and EMG signal patterns during repeated bouts of concentric or eccentric muscle actions. Acta Physiol Scand 1990;138:263-71.

22. Durand RJ, Castracane VD, Hollander DB, et al. Hormonal responses from concentric and eccentric muscle contractions. Med Sci Sports Exerc 2003;35:937-43.

23. Váczi M, Río-Rodríguez D, Négyesi J, et al. Acute neuromechanical modifications and 24-h recovery in quadriceps muscle after maximal stretch-shortening cycle exercise. J Electromyogr Kinesiol 2018;40:64-71.

24. Vincent HK, Vincent KR. The effect of training status on the serum creatine kinase response, soreness and muscle function following resistance exercise. Int J Sports Med 1997;18:431-7.

25. Gorianovas G, Skurvydas A, Streckis V, et al. Repeated bout effect was more expressed in young adult males than in elderly males and boys. Biomed Res Int 2013;2013:218970.

26. Klapcińska B, Iskra J, Poprzecki S, et al. The effects of sprint $(300 \mathrm{~m})$ running on plasma lactate, uric acid, creatine kinase and lactate dehydrogenase in competitive hurdlers and untrained men. J Sports Med Phys Fitness 2001;41:306-11.

27. Costill DL, Daniels J, Evans W, et al. Skeletal muscle enzymes and fiber composition in male and female track athletes. J Appl Physiol 1976;40:149-54.

28. Di Mauro D, Currò M, Trimarchi F, et al. Role of Genetic Background in Cardiovascular Risk Markers Changes in Water Polo Players. Int J Sports Med 2018;39:390-6.

29. Carmona G, Mendiguchía J, Alomar X, et al. Time Course and Association of Functional and Biochemical Markers in Severe Semitendinosus Damage Following Intensive Eccentric Leg Curls: Differences between and within Subjects. Front Physiol 2018;9:54.

30. Nagy SA, Váczi M, Perlaki G, et al. Acute and delayed effects of concentric and eccentric exercise on quantitative muscle MRI. Acta Radiol 2019;60:902-7. 